\title{
Dosimetric coverage of the prostate, normal tissue sparing, and acute toxicity with high-dose-rate brachytherapy for large prostate volumes
}

\author{
George Yang ${ }^{1}$, Tobin J. Strom ${ }^{1}$, Richard B. Wilder ${ }^{2}$, Kushagra Shrinath ${ }^{1}$, Eric A. Mellon ${ }^{1}$, Daniel C. \\ Fernandez ${ }^{1}$, Matthew C. Biagioli ${ }^{1}$ \\ ${ }^{1}$ Department of Radiation Oncology, H. Lee Moffitt Cancer Center and Research Institute, Tampa, FL \\ 33612, USA; ${ }^{2}$ Cancer Treatment Centers of America, Newnan, GA 30265, USA
}

\section{ABSTRACT}

Purpose: To evaluate dosimetric coverage of the prostate, normal tissue sparing, and acute toxicity with HDR brachytherapy for large prostate volumes.

Materials and Methods: One hundred and two prostate cancer patients with prostate volumes $>50 \mathrm{~mL}$ (range: 5-29 mL) were treated with high-dose-rate (HDR) brachytherapy \pm intensity modulated radiation therapy (IMRT) to 4,500 cGy in 25 daily fractions between 2009 and 2013. HDR brachytherapy monotherapy doses consisted of two 1,350-1,400 cGy fractions separated by 2-3 weeks, and HDR brachytherapy boost doses consisted of two 950-1,150 cGy fractions separated by 4 weeks. Twelve of 32 (38\%) unfavorable intermediate risk, high risk, and very high risk patients received androgen deprivation therapy. Acute toxicity was graded according to the Common Terminology Criteria for Adverse Events (CTCAE) version 4.

Results: Median follow-up was 14 months. Dosimetric goals were achieved in over 90\% of cases. Three of $102(3 \%)$ patients developed Grade 2 acute proctitis. No variables were significantly associated with Grade 2 acute proctitis. Seventeen of 102 (17\%) patients developed Grade 2 acute urinary retention. American Urological Association (AUA) symptom score was the only variable significantly associated with Grade 2 acute urinary retention $(\mathrm{p}=0.04)$. There was no $\geq$ Grade 3 acute toxicity.

Conclusions: Dosimetric coverage of the prostate and normal tissue sparing were adequate in patients with prostate volumes $>50 \mathrm{~mL}$. Higher pre-treatment AUA symptom scores increased the relative risk of Grade 2 acute urinary retention. However, the overall incidence of acute toxicity was acceptable in patients with large prostate volumes.

\section{ARTICLE INFO}

Key words:

Iridium; Brachytherapy; Prostatic Neoplasms; Radiometry; Urinary Retention

Int Braz J Urol. 2015; 41: 435-41

Submitted for publication: June 12, 2014

Accepted after revision: August 10, 2014

\section{INTRODUCTION}

Use of high-dose-rate brachytherapy (HDR) for prostate cancer has increased over the last decade (1). HDR brachytherapy has produced excellent outcomes as either monotherapy (2) or as a boost in combination with external beam radiation therapy (EBRT) $(3,4)$.
The Groupe Européen de Curietherapie (GEC) European Society for Radiotherapy and Oncology (ESTRO)-European Association of Urology (EAU) consider prostate volumes larger than $60 \mathrm{~mL}$ to be less suitable for HDR brachytherapy due to increased pubic arch interference and concerns regarding greater toxicity (5). Similarly, the American Brachytherapy Society has su- 
ggested that prostate volumes greater than 50 $\mathrm{mL}$ constitute a relative contraindication to HDR brachytherapy (6).

The purpose of this study is to evaluate dosimetric coverage of the prostate, normal tissue sparing, and acute toxicity with HDR brachytherapy in patients with prostate volumes $>50 \mathrm{~mL}$.

\section{MATERIALS AND METHODS}

Patient Characteristics

Patient records were reviewed after institutional review board approval was obtained. Patients were included in this study if they had clinically localized prostate cancer, prostate volumes greater than $50 \mathrm{~mL}$, and underwent HDR brachytherapy without or with intensity modulated radiation therapy (IMRT) at a single institution between 2009 and 2013. Prostate volume was calculated using pre-treatment computed tomography (CT) scans. The mean \pm standard deviation prostate volume was $68 \pm 16 \mathrm{~mL}$ (range, 51 $129 \mathrm{~mL}$ ). National Comprehensive Cancer Network (NCCN) definitions of very low, low, intermediate, high, and very high recurrence risk groups were used (7). Patient characteristics are presented in Table-1. All patients received alpha blockers for a minimum of one month after HDR brachytherapy.

\section{HDR Brachytherapy Monotherapy}

Prostate cancer patients with very low risk disease and an expected survival of $\geq 20$ years, low

Table 1 - Patient characteristics.

\begin{tabular}{lcc}
\hline Patient Characteristics & Category & Number (\%) \\
\hline Age (years) & Median & 69 \\
Gleason Score & $\leq 6$ & $38(37)$ \\
& $78(47)$ \\
Recurrence Risk Group & Very Low to Low & $16(16)$ \\
& Intermediate & $34(33)$ \\
Pre-treatment Prostate-Specific Antigen (ng/mL) & High to Very High & $47(46)$ \\
& $<10$ & $21(21)$ \\
Prostate Volume (mL) & $10-20$ & $82(80)$ \\
Body Mass Index & $>20$ & $12(12)$ \\
Androgen Deprivation Therapy & Median & $8(8)$ \\
Pre-treatment American Urological Association Symptom & Median & 62 \\
Score & No & 28.7 \\
& Yes & 86 \\
& $20-35$ (Severe Symptoms) & 16
\end{tabular}


risk disease and an expected survival of $\geq 10$ years, or "favorable" intermediate risk disease were treated with HDR brachytherapy monotherapy to the prostate. "Favorable" intermediate risk patients were defined as those with a Gleason score $3+4=7$, American Joint Committee on Cancer clinical $\leq \mathrm{T} 2 \mathrm{~b}$ disease (8), and $\leq 50 \%$ positive core biopsies (2).

Patients underwent HDR brachytherapy monotherapy according to American Brachytherapy Society consensus guidelines (6). Patients were placed under general anesthesia, put in the low dorsal lithotomy position, and prepared and draped in sterile fashion. A \# 16 Foley catheter was inserted into the bladder and $5 \mathrm{~mL}$ of diatrizoate contrast was injected into the catheter balloon. A biplane trans-rectal ultrasound (TRUS) probe was inserted into the rectum and mounted on an adjustable stepper stabilizer. A prostate template was attached to the front of the TRUS stabilizer unit.

Fourteen to eighteen ProGuide ${ }^{\mathrm{TM}}$ (Nucletron, Veenendaal, Netherlands) HDR brachytherapy treatment catheters were inserted into the prostate transperineally under TRUS guidance. Each treatment catheter was carefully advanced so as to not penetrate the inferior bladder wall or come within $1.0 \mathrm{~cm}$ of the prostatic urethra. Once the treatment catheters were in position, the prostate template was sutured onto the perineum and locked. Upon completion of treatment catheter placement, the prostate template was removed.

The clinical target volume (CTV) consisted of the prostate as defined by CT scan. The planning target volume (PTV) was the same as the CTV. The PTV was treated with two 1,350-1,400 cGy fractions. One fraction was delivered with each iridium-192 (Ir-192) implant, and the two implants were separated by 2-3 weeks. Prostate doses were recorded as the minimum dose that covered more than $90 \%$ of the prostate volume expressed as a percentage of the prescription dose (prostate D90). Prostate volumes were recorded as the fractional volume of the prostate that received $100 \%$ of the prescribed dose (prostate V100), 150\% of the prescribed dose (prostate V150), and 200\% of the prescribed dose (prostate V200). The urethral volume was recorded as the fractional volume of the urethra that received $150 \%$ of the prescribed dose (urethra V150). The urethral dose was the dose de- livered to $10 \%$ of the urethral volume expressed as a percentage of the prescribed dose (urethra D10). The rectal volume was recorded as the fractional volume of the rectum that received $75 \%$ of the prescribed dose (rectum V75). Rectal doses were recorded as the maximum dose received by $2 \mathrm{~mL}$ of the rectum expressed as a percentage of the prescribed dose (rectal D2 mL). The bladder volume was recorded as the fractional volume of the bladder that received $75 \%$ of the prescribed dose (bladder V75). Bladder doses were expressed as the maximum dose received by $2 \mathrm{~mL}$ of the bladder expressed as a percentage of the prescribed dose (bladder D2 $\mathrm{mL}$ ). Dosimetric goals are presented in Table-2. No androgen deprivation therapy (ADT) was given to HDR brachytherapy monotherapy patients, e.g., to down-size the prostate.

\section{HDR Brachytherapy + IMRT \pm ADT}

"Unfavorable" intermediate risk patients had a Gleason score $4+3=7$, clinical T2c disease, or $>50 \%$ positive core biopsies $(9,10)$. Unfavorable intermediate risk and high risk patients were treated with an HDR brachytherapy boost consisting of two 950-1,150 cGy fractions and IMRT to the prostate and proximal $1.0 \mathrm{~cm}$ of the seminal vesicles to 4,500 cGy in 25 fractions over 5 weeks without or with ADT (4). The two HDR brachytherapy fractions were delivered $11 / 2$ weeks before the start of IMRT and $21 / 2$ weeks after the start of the IMRT. The CTV for each HDR brachytherapy fraction consisted of the prostate as defined by CT scan. In cases with extraprostatic extension on MRI or biopsy, the CTV was expanded to include disease outside of the prostate with a $0.3 \mathrm{~cm}$ margin. In cases with seminal vesicle invasion on MRI, the CTV was expanded to the seminal vesicle involvement. The PTV was the same as the CTV. Four fiducial gold markers were transrectally inserted into the left and right mid lateral prostate and the prostatic base and apex at the time of the first HDR brachytherapy implant. The markers made it possible to determine the location of the prostate using electronic portal imaging immediately prior to each IMRT treatment and thereby deliver image-guided radiation therapy (11). No IMRT was delivered the day of the second HDR brachytherapy fraction. 
Table 2 - Dosimetric results achieved with HDR brachytherapy.

\begin{tabular}{lcccc}
\hline Dosimetric Endpoint & Goal & Achieved Mean & Achieved Standard Deviation & Achieved Range \\
\hline Prostate & $>90$ and $<130$ & 105 & 3 & $87-111$ \\
D90 (\%) & $>90$ & 94 & 2 & $87-98$ \\
V100 (\%) & $<50$ & 27 & 3 & $20-36$ \\
V150 (\%) & $<25$ & 11 & 2 & $7-22$ \\
V200 (\%) & 0 & & & \\
Urethra & $<115$ & 108 & 0.0 & $0.0-0.0$ \\
V150 (\%) & & & 2 & $105-120$ \\
D10 (\%) & $<1.0$ & 0.2 & 0.3 & $0.0-1.5$ \\
Rectum & $<75$ & 54 & 10 & $33-75$ \\
V75 (mL) & & & \\
D2 $\mathrm{mL}(\%)$ & $<1.0$ & 0.7 & 0.5 & $0.0-3.0$ \\
Bladder & $<80$ & 65 & 6 & $45-79$ \\
V75 $(\mathrm{mL})$ & & & & \\
D2 $\mathrm{mL}(\%)$ &
\end{tabular}

Patients were simulated for IMRT with an empty rectum using a pelvic CT scan with $0.3 \mathrm{~cm}$ slices. Forty $\mathrm{mL}$ normal saline mixed with $10 \mathrm{~mL}$ non-ionic contrast was injected into the bladder and urethra at the time of simulation in order to perform a cystogram and urethrogram, respectively. The CTV for IMRT consisted of the prostate and inferomedial $1.0 \mathrm{~cm}$ of the seminal vesicles as defined by $\mathrm{CT}$ and magnetic resonance imaging (MRI) scans. In cases with extraprostatic extension on MRI or biopsy, the CTV for IMRT was expanded to include disease outside of the prostate based on CT-MRI fusion. In cases with seminal vesicle invasion on MRI, the CTV for IMRT was expanded to include the entire involved seminal vesicle. The PTV consisted of the CTV with $0.5 \mathrm{~cm}$ expansion posteriorly, inferiorly, and superiorly and $0.7 \mathrm{~cm}$ expansion anteriorly and laterally. The minimum allowable dose delivered to the PTV was 93\% of the prescribed dose, and the maximum allowable dose delivered to the PTV was 115\% of the prescribed dose. At least $98 \%$ of the PTV received $\geq 95 \%$ of the prescribed dose (12). Rectal and bladder dose-volume histograms (DVHs) were created. The dosimetric goals for IMRT were that no more than $15 \%$ of the rectal volume should receive $>4,100$ cGy and no more than 15\% of the bladder volume should receive $>4,000 \mathrm{cGy}$.

\section{Biologically Effective Doses $\left(\mathrm{BED}_{1.5}\right)$}

Assuming an $\alpha / \beta$ ratio of 1.5 for prostate cancer (13), the biologically effective doses $\left(\mathrm{BED}_{1.5}\right)$ with HDR brachytherapy monotherapy to a total dose of 2,700-2,800 cGy in 2 fractions versus an HDR brachytherapy boost to $1,900-2,300$ cGy in 2 fractions in combination with IMRT to 4,500 cGy in 25 fractions are 27,000-28,933 cGy, and 23,833-29,833 cGy, respectively.

\section{ADT}

ADT always consisted of a gonadotropin-releasing hormone agonist. In most cases, ADT also included an anti-androgen. The median duration of a gonadotropin-releasing hormone agonist was 4 months in a neoadjuvant and concomitant setting for intermediate-risk disease and 28 months in a neoadjuvant, concomitant, and adjuvant setting for high risk or very high risk 
disease. The median duration of an anti-androgen was one month starting two weeks prior to the gonadotropin-releasing hormone agonist. Twelve of $32(38 \%)$ unfavorable intermediate risk, high risk, and very high risk patients received ADT. ADT use in these cases was at the discretion of the patients and began two months prior to the start of radiotherapy. None of the patients who received ADT had a history of congestive heart failure or myocardial infarction.

\section{Acute Toxicity}

The severity of adverse effects was graded according to the Common Terminology Criteria for Adverse Events version 4.0 (14).

\section{Statistics}

Statistical analysis was performed using Minitab ${ }^{\circledR}$ Statistical Software version 16 (Minitab $^{\circledR}$, Inc, State College, PA). A two sided $\mathrm{p} \leq 0.05$ was considered significant. Means, standard deviations, and minimum and maximum values of the dosimetric parameters were calculated. Multivariate logistic regression analysis was used to assess the association between prostate volume, body mass index (BMI), pre-treatment American Urological Association (AUA) symptom score, total HDR dose, dosimetric parameters (D90, V100) and the occurrence of $\geq$ Grade 2 acute urinary retention and acute proctitis.

\section{RESULTS}

Median follow-up was 14 months. Dosimetric results achieved with HDR brachytherapy are presented in Table- 2 . In terms of prostate coverage and sparing of the urethra, rectum, and bladder, dosimetric goals were achieved in over $90 \%$ of cases.

Three of 102 (3\%) patients developed Grade 2 acute proctitis. Seventeen of 102 (17\%) patients developed Grade 2 acute urinary retention requiring placement of a Foley catheter for less than 6 weeks. Only AUA symptom score was significantly associated, on multivariate analysis, with Grade 2 acute urinary retention (odds ratio 1.14, 95\% confidence interval 1.01-1.28, $\mathrm{p}=0.04)$. No variables were significantly associated with Grade 2 acute proctitis. There was no $\geq$ Grade 3 acute toxicity.

\section{DISCUSSION}

Monroe et al. (15) performed a study of 54 patients who received either HDR brachytherapy monotherapy or HDR brachytherapy as a boost to EBRT. Large prostate volumes did not affect dosimetric coverage of the prostate, with prostate D90, V100 and V150 meeting the dosimetric goals. In addition, genitourinary and gastrointestinal toxicity in patients with prostates $>50 \mathrm{~mL}$ was consistent with previously-reported toxicity for small prostates $(6,16)$. Le et al. (17) performed a study of 164 prostate cancer patients who underwent HDR brachytherapy monotherapy and found no significant difference in prostate D90 or V100 with prostate volumes $>50 \mathrm{~mL}$. In patients with larger glands, a significantly higher biochemical control of disease was observed, with no difference in late toxicity. They concluded that gland size should not be considered in the selection of patients for HDR brachytherapy. White et al. (18) reported that HDR brachytherapy allows the physician to consistently achieve complete prostate target coverage and maintain normal tissue dose constraints for organs at risk over target volumes ranging from 12-109 mL. Similarly, in this study, prostate coverage and sparing of the urethra, rectum, and bladder were dosimetrically achieved in over 90\% of cases with prostate volumes ranging between $51 \mathrm{~mL}$ and $129 \mathrm{~mL}$ (Table-2).

In this report, ADT was only given to $12 / 32(38 \%)$ unfavorable intermediate risk, high risk, and very high risk patients. Neoadjuvant ADT may be used to down-size an enlarged prostate due to benign prostatic hyperplasia (BPH) regardless of the recurrence risk group $(6,19)$. However, ADT has been implicated in brachytherapy-related morbidity such as urinary retention (20). Also, there may be a significant detriment in quality of life due to ADT (21).

In the present report, the incidence rate of Grade 2 acute urinary retention was $17 \%$. This is consistent with the literature (22). There was an independent association on multivariate analysis between pre-treatment AUA symptom score and 
acute urinary retention. An increased pre-treatment AUA symptom score may be due to BPH (21). Some groups have reported that high AUA symptom scores are associated with an increased risk of urinary retention $(24,25)$. Similar findings were observed in this study. AUA symptom scores improve after brachytherapy significantly faster in patients receiving alpha blockers (26).

A brachytherapy boost offers a potential radiobiological benefit over conventionally-fractionated IMRT by delivering hypofractionated treatment, which may increase the sensitivity of prostate cancer to radiation therapy by favorably affecting the $\alpha / \beta$ ratio (27-29). This may explain why some have observed improved biochemical disease-free survival with IMRT and a brachytherapy boost compared with IMRT alone, though there is a greater risk of late genitourinary toxicity (30). There was no $\geq$ Grade 3 acute toxicity due to an HDR brachytherapy boost and IMRT without or with ADT in the present study.

Limitations of this study are primarily related to its retrospective nature, limited number of patients, and short follow-up. Nevertheless, this is one of the few reports on HDR brachytherapy in men with large prostates.

\section{CONCLUSIONS}

Prostate volumes greater than $50 \mathrm{~mL}$ do not constitute a relative contraindication to HDR brachytherapy.

\section{CONFLICT OF INTEREST}

None declared.

\section{REFERENCES}

1. Zaorsky NG, Doyle LA, Yamoah K, Andrel JA, Trabulsi EJ, Hurwitz MD,et al. High dose rate brachytherapy boost for prostate cancer: a systematic review. Cancer Treat Rev. 2014:40:414-25.

2. Ghilezan M, Martinez A, Gustason G, Krauss D, Antonucci JV, Chen P,et al. High-dose-rate brachytherapy as monotherapy delivered in two fractions within one day for favorable/ intermediate-risk prostate cancer: preliminary toxicity data. Int J Radiat Oncol Biol Phys. 2012;83:927-32.
3. Deutsch I, Zelefsky MJ, Zhang Z, Mo Q, Zaider M, Cohen G, et al. Comparison of PSA relapse-free survival in patients treated with ultra-high-dose IMRT versus combination HDR brachytherapy and IMRT. Brachytherapy. 2010;9:313-8.

4. Martinez AA, Gonzalez J, Ye H, Ghilezan M, Shetty S, Kernen $\mathrm{K}$, et al. Dose escalation improves cancer-related events at 10 years for intermediate- and high-risk prostate cancer patients treated with hypofractionated high-dose-rate boost and external beam radiotherapy. Int J Radiat Oncol Biol Phys. 2011;79:363-70.

5. Hoskin PJ, Colombo A, Henry A, Niehoff P, Paulsen Hellebust T, Siebert FA, et al. GEC/ESTRO recommendations on high dose rate afterloading brachytherapy for localised prostate cancer: an update. Radiother Oncol. 2013;107:325-32.

6. Yamada Y, Rogers L, Demanes DJ, Morton G, Prestidge BR, Pouliot J, et al. American Brachytherapy Society. American Brachytherapy Society consensus guidelines for high-doserate prostate brachytherapy. Brachytherapy. 2012;11:20-32.

7. Mohler JL, Armstrong AJ, Bahnson RR, Boston B, Busby JE, D'Amico AV, et al. Prostate cancer, Version 3.2012: featured updates to the NCCN guidelines. J Natl Compr Canc Netw. 2012;10:1081-7.

8. Edge SB, Compton CC. The American Joint Committee on Cancer: the 7th edition of the AJCC cancer staging manual and the future of TNM. Ann Surg Oncol. 2010;17:1471-4.

9. Castle KO, Hoffman KE, Levy LB, Lee AK, Choi S, Nguyen $\mathrm{QN}$, et al. Is androgen deprivation therapy necessary in all intermediate-risk prostate cancer patients treated in the dose escalation era? Int J Radiat Oncol Biol Phys. 2013;85:693-9.

10. Huang J, Vicini FA, Williams SG, Ye H, McGrath S, Ghilezan $M$, et al. Percentage of positive biopsy cores: a better risk stratification model for prostate cancer? Int J Radiat Oncol Biol Phys. 2012;83:1141-8.

11. Vetterli D, Thalmann S, Behrensmeier F, Kemmerling L, Born EJ, Mini R, et al. Daily organ tracking in intensity-modulated radiotherapy of prostate cancer using an electronic portal imaging device with a dose saving acquisition mode. Radiother Oncol. 2006;79:101-8.

12. Shaffer R, Morris WJ, Moiseenko V, Welsh M, Crumley C, Nakano S, et al. Volumetric modulated Arc therapy and conventional intensity-modulated radiotherapy for simultaneous maximal intraprostatic boost: a planning comparison study. Clin Oncol (R Coll Radiol). 2009;21:401-7.

13. Fowler JF. The radiobiology of prostate cancer including new aspects of fractionated radiotherapy. Acta Oncol. 2005;44:265-76.

14. National Cancer Institute. Common Terminology Criteria for Adverse Events v4.0. NIH publication \#09-7473. Published on May 28, 2009. Retrieved on June 14, 2010 Available at: http:// evs.nci.nih.gov/ftp1/CTCAE/CTCAE_4.03_2010-06-14_ QuickReference_5x7.pdf 
15. Monroe AT, Faricy PO, Jennings SB, Biggers RD, Gibbs GL, Peddada AV. High-dose-rate brachytherapy for large prostate volumes (> or $=50 \mathrm{cc}$ )-Uncompromised dosimetric coverage and acceptable toxicity. Brachytherapy. 2008;7:7-11.

16. Zelefsky MJ, Levin EJ, Hunt M, Yamada Y, Shippy AM, Jackson $A$, et al. Incidence of late rectal and urinary toxicities after threedimensional conformal radiotherapy and intensity-modulated radiotherapy for localized prostate cancer. Int J Radiat Oncol Biol Phys. 2008;70:1124-9.

17. Le H, Rojas A, Alonzi R, Hughes R, Ostler P, Lowe G, et al. The influence of prostate volume on outcome after high-dose-rate brachytherapy alone for localized prostate cancer. Int J Radiat Oncol Biol Phys. 2013;87:270-4.

18. White EC, Kamrava MR, Demarco J, Park SJ, Wang PC, Kayode 0 , et al. High-dose-rate prostate brachytherapy consistently results in high quality dosimetry. Int J Radiat Oncol Biol Phys. 2013;85:543-8.

19. Kovács G, Pötter R, Loch T, Hammer J, Kolkman-Deurloo IK, de la Rosette JJ,et al. GEC/ESTRO-EAU recommendations on temporary brachytherapy using stepping sources for localised prostate cancer. Radiother Oncol. 2005;74:137-48.

20. Merrick GS, Wallner KE, Butler WM. Patient selection for prostate brachytherapy: more myth than fact. Oncology (Williston Park). 2004;18:445-52; discussion 452, 455-7.

21. Gay HA, Michalski JM, Hamstra DA, Wei JT, Dunn RL, Klein EA, et al. PROSTQA Consortium. Neoadjuvant androgen deprivation therapy leads to immediate impairment of vitality/ hormonal and sexual quality of life: results of a multicenter prospective study. Urology. 2013;82:1363-8.

22. Demanes DJ, Martinez AA, Ghilezan M, Hill DR, Schour L, Brandt $D$, et al. High-dose-rate monotherapy: safe and effective brachytherapy for patients with localized prostate cancer. Int J Radiat Oncol Biol Phys. 2011;81:1286-92.
23. McVary KT, Roehrborn CG, Avins AL, Barry MJ, Bruskewitz RC, Donnell RF, et al. Update on AUA guideline on the management of benign prostatic hyperplasia. J Urol. 2011;185:1793-803.

24. Roeloffzen EM, Hinnen KA, Battermann JJ, Monninkhof EM, van Roermund JG, van Gellekom MP, et al. The impact of acute rinary retention after iodine-125 prostate brachytherapy on health-related quality of life. Int J Radiat Oncol Biol Phys. 2010;77:1322-8.

25. Schwartz DJ, Schild SE, Wong WW, Vora SA. Factors associated with the frequency of self-intermittent catheterization after prostate brachytherapy. Int $\mathrm{J}$ Radiat Oncol Biol Phys. 2005;61:60-3.

26. Elshaikh MA, Ulchaker JC, Reddy CA, Angermeier KW, Klein EA, Chehade N, et al. Prophylactic tamsulosin (Flomax) in patients undergoing prostate $125 \mathrm{I}$ brachytherapy for prostate carcinoma: final report of a double-blind placebocontrolled randomized study. Int J Radiat Oncol Biol Phys. 2005;62:164-9.

27. Brenner DJ, Martinez AA, Edmundson GK, Mitchell C, Thames HD, Armour EP. Direct evidence that prostate tumors show high sensitivity to fractionation (low alpha/beta ratio), similar to late-responding normal tissue. Int J Radiat Oncol Biol Phys. 2002;52:6-13.

28. Martinez AA, Demanes J, Vargas C, Schour L, Ghilezan M, Gustafson GS. High-dose-rate prostate brachytherapy: an excellent accelerated-hypofractionated treatment for favorable prostate cancer. Am J Clin Oncol. 2010;33:481-8.

29. Wong WM, Wallner KE. The case for hypofractionation of localized prostate cancer. Rev Urol. 2013;15:113-7.

30. Bannuru RR, Dvorak T, Obadan N, Yu WW, Patel K, Chung $\mathrm{M}$, et al. Comparative evaluation of radiation treatments for clinically localized prostate cancer: na updated systematic review. Ann Intern Med. 2011;155:171-8.
Correspondence address: Matthew C. Biagioli, MD

Florida Hospital Cancer, Institute Department of Radiation Oncology 2600 Westhall Lane Maitland, FL 32751, USA Telephone: +1 305 978-4617 E-mail: mcbiagioli@yahoo.com 